\title{
Certain Results on Hypergeometric Series for Meijer's G-Functions
}

\author{
Basanta Raj Sharma \\ Department of Mathematics, Mahendra M. Campus T.U. Dang \\ e-mail: basantrs@yahoo.com
}

\begin{abstract}
This paper has deal with certain aspects in the development of hypergeometric series, which are the most significant topics of special function. Moreover, making use of the known results, Meijer's G-functions of hypergeometric type have also been generalized by increasing the number of variables in terms of double Barnes' type of contour integrals.
\end{abstract}

Key words: hypergeometric series, Meijer's G-function, $q$-series, the ${ }_{p} F_{q}$-series, when $p>q+1$, Mellin-Barnes' type, double summation

\subsection{Introduction}

Generalized hypergeometric series, both ordinary and basic have been a very significant tool in the definition of Meijer's G-functions. In what follows, the usual hypergeometric notation and definition shall be followed throughout.

Let $[\mathrm{a}]_{\mathrm{n}}=\mathrm{a}(\mathrm{a}+1)(\mathrm{a}+2) \ldots \ldots(\mathrm{a}+\mathrm{n}-1), \mathrm{n} \geq 1$,

(a) ${ }_{0}=1$, then the generalized hypergeometric series is defined by

$$
\begin{aligned}
& \text { (1) }{ }_{\mathrm{r}} \mathrm{F}_{\mathrm{s}}\left[\begin{array}{l}
\left.a_{1}, a_{2}, \ldots \ldots \ldots, a_{y} ; z\right] \\
b_{1}, b_{2}, \ldots \ldots \ldots \ldots, b_{s}
\end{array}\right]{ }_{=\mathrm{r}} \mathrm{F}_{\mathrm{s}}\left[\left(\mathrm{a}_{\mathrm{r}}\right) ;\left(\mathrm{b}_{\mathrm{s}}\right) ; \mathrm{z}\right] \\
& =\sum_{n=0}^{\infty} \frac{\left(a_{1}\right)_{n}\left(a_{2}\right)_{n} \ldots \ldots\left(a_{y}\right)_{n}}{\left(b_{1}\right)_{n}\left(b_{2}\right)_{n} \ldots \ldots\left(b_{s}\right)_{n}} \cdot \frac{z^{n}}{(1)_{n}} \\
& =\sum_{n=0}^{\infty} \frac{\left[\left(a_{y}\right)\right]_{n} z^{n}}{\left[\left(b_{s}\right)\right]_{n}(1)_{n}}
\end{aligned}
$$$$
\text { (iii) diverges for } \mathrm{z} \neq 0 \text { if } \mathrm{r}>\mathrm{s}+1
$$

If $\mathrm{r}=\mathrm{s}+1$, the series converges absolutely on the

circle $|\mathrm{z}|=1$ when

$\mathrm{R}_{\mathrm{e}}\left(\sum_{0}^{s} b_{s}-\sum_{1}^{r} a_{r}\right)>0$

Also, when $r=s+1$, the series is called

"Saalschutzian" if $\mathrm{R}_{\mathrm{e}}\left(\sum_{0}^{s} b_{s}-\sum_{1}^{r} a_{r}\right)=1$ and

"Well-poised" if $1+a_{1}=b_{1}+a_{2}=b_{2}+a_{3}=\ldots \ldots$.

$$
=\mathrm{b}_{\mathrm{s}}+\mathrm{a}_{\mathrm{s}+1}
$$

If any one of the numerator parameters in (1) is zero or a negative integer, $\mathrm{F}_{\mathrm{s}}$ reduces to a polynomial, but if any $\mathrm{b}$ parameter is a negative integer, $-\mathrm{N}$, say, where $\mathrm{N}=1,2 \ldots \ldots$ (unless any of the parameters is also a negative integer $-\mathrm{M}$, say, where $\mathrm{M}=\mathrm{N}$, $\mathrm{N}+1, \ldots \ldots$, or zero), then $\mathrm{F}_{\mathrm{s}}$ function is not defined.

where $\left(a_{r}\right)$ stand for $r$ parameters $a_{1}, a_{2}, \ldots . ., a_{r}$ and so for $\left(b_{s}\right)$. The series $1.2(1)$ converges

In case of the basic or q-hypergeometric series

(i) for all finite $\mathrm{z}$ if $\mathrm{r} \leq \mathrm{s}$,

(ii) for $|\mathrm{z}|<1$ if $\mathrm{r}=\mathrm{s}+1$, and for any positive integer $n$, let $\left[a ; q^{k}\right]_{n}=(1-a)(1-$ $\left.a q^{k}\right) \ldots . .\left(1-\mathrm{aq}^{\mathrm{k}(\mathrm{n}-1)}\right), \mathrm{n} \geq 1$, 
$\left[\mathrm{a} ; \mathrm{q}^{\mathrm{k}}\right]_{0}=1,\left[\mathrm{a} ; \mathrm{q}^{\mathrm{k}}\right]_{\mathrm{N}}=\prod_{n=0}^{N}\left(1-a q^{k}\right),|\mathrm{q}|<1$,

A generalized basic or q-hypergeometric series is defined by

(2) ${ }_{\mathrm{r}} \phi_{\mathrm{s}}\left[\mathrm{a}_{1} \ldots \ldots, \mathrm{a}_{\mathrm{r}} ; \mathrm{b}_{1} \ldots . ., \mathrm{b}_{\mathrm{s}} ; \mathrm{q}^{\mathrm{k}} ; \mathrm{z}\right]$

$={ }_{\mathrm{r}} \phi_{\mathrm{s}}\left[\left(\mathrm{a}_{\mathrm{r}}\right) ;\left(\mathrm{b}_{\mathrm{s}}\right) ; \mathrm{q}^{\mathrm{k}} ; \mathrm{z}\right]$

$=\sum_{n=0}^{\infty} \frac{\left[a_{1} ; q^{k}\right]_{n} \ldots \ldots\left[a_{r} ; q^{k}\right]_{n} z^{n}}{\left[q^{k} ; q^{k}\right]_{n}\left[b_{1} ; q^{k}\right]_{n} \ldots \ldots\left[b_{s} ; q^{k}\right]_{n}}(-)^{\mathrm{n}} \mathrm{q}^{\{\mathrm{kn}(\mathrm{n}-1) /}$ 2)(1+s-r)

where $\mathrm{q} \neq 0$ and $\mathrm{r}<\mathrm{s}+1$.

The series converges absolutely for all finite $z$, if $\mathrm{r} \leq \mathrm{s}$ and for $|\mathrm{z}|<1$ if

$r=s+1$. When $r=s+1,(2)$ is said to be "Saalschutzain" if

$b_{1} b_{2} \ldots \ldots b_{s}=q^{k} a_{1} a_{2} \ldots \ldots a_{s+1}$

and "Well-poised" if

$\mathrm{q}^{\mathrm{k}} \mathrm{a}_{1}=\mathrm{b}_{1} \mathrm{~b}_{2}=\mathrm{b}_{\mathrm{s}} \mathrm{a}_{\mathrm{s}+1 .]}$

Whenever there is no chance of confusion and $\mathrm{k}=1$, we shall omit it from all symbols.

A Kampç dç Fçriçt hypergeometric function of two variables is defined as :

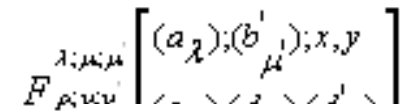

$$
\begin{aligned}
& =\sum_{m=0}^{\infty} \sum_{n=0}^{\infty} \frac{\left[\left(a_{\lambda}\right)\right]_{m+n}\left[\left(b_{\mu}\right)\right]_{m}\left[\left(b_{\mu}\right)\right]_{n} x^{m} y^{n}}{\left[\left(c_{\rho}\right)\right]_{m+n}\left[\left(d_{\nu}\right)\right]_{m}\left[\left(b_{\nu}\right)\right]_{N}(1)_{m}(1)_{n}}
\end{aligned}
$$

where $\left(a_{\lambda}\right)_{\mathrm{n}}$ stand for the sequence of parameters $\mathrm{a}_{1}$, $a_{2}, \ldots \ldots \ldots, \ldots, x \cdot<1, . y \cdot<1$ and $++'<+++^{\prime}+1$ for convergence.

\section{Meijer's G-functions}

C.S. Meijer (1936) provided an interpolation of symbol $\mathrm{F}_{\mathrm{q}}$ for $\mathrm{p}>\mathrm{q}+1$ which is in complete agreement with Mac-Robert's E-function. He defined G-function as a sum of $\underset{\mathrm{p}}{\mathrm{F}} \mathrm{q}$ series and later on replaced this definition in terms of Mellin-Barnes' type of contour integrals.
The latter definition has the advantages in the sense that it allows a greater freedom in the relative values of $p$ and q. The G-function of one variable is thus defined as

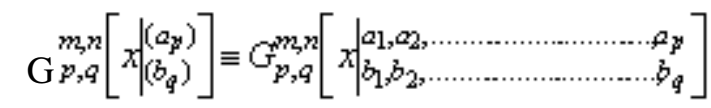

$$
\begin{aligned}
& =\frac{1}{2 \pi i} \int_{L} \Gamma\left[\begin{array}{l}
\left(b_{m}\right)-\xi, 1-\left(a_{n}\right)+\xi ; \\
1-\left(b_{m+1, q}\right)+\xi,\left(a_{n+1, p}\right)-\xi
\end{array}\right] x^{\xi} d \xi \\
& \equiv \frac{1}{2 \pi} \int_{L} \frac{\prod_{j=1}^{m} \Gamma\left(b_{j}-\xi\right) \prod_{j=1}^{n} \Gamma\left(1-a_{j}+\xi\right)}{\prod_{j=+1}^{q} \Gamma\left(1-b_{j}+\xi\right) \prod_{j=1+1}^{y} \Gamma\left(a_{j}-\xi\right)} x^{5} d \xi,
\end{aligned}
$$

where an empty product is interpreted as unity; p, q, $\mathrm{m}, \mathrm{n}$ are non-negative integers satisfying $0 \leq \mathrm{m} \leq \mathrm{q}, 0$ $\leq \mathrm{n} \leq \mathrm{p}$ and the parameters are such that no pole of $\Gamma\left(b_{j}-\xi\right), j=1, \ldots \ldots, m$, coincides with any pole of $\tilde{\mathrm{A}}\left[1-a_{j}+\xi\right), j=1, \ldots \ldots, n$. Whenever there is no danger of misunderstanding, it shall be written more briefly as

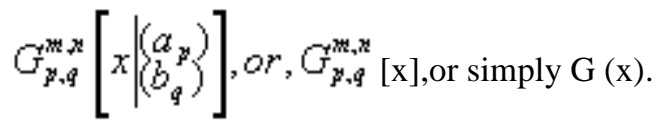

The integral (4) converges if $n+q<2(m+n)$ and $|\arg x|<\pi\left[m+n-\frac{1}{2}(p+q)\right]$.

\subsection{G-function of one variable}

We shall now establish the following results involving G-function of one variable. 
(1)

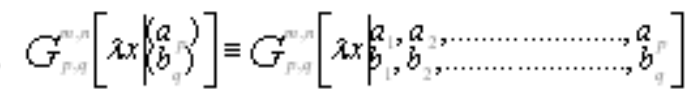

$=\lambda^{4} \sum_{=0}^{\infty} \frac{1}{(1)}(1-\lambda) G_{P, Q}^{m, Q}\left[\begin{array}{l}x_{1}, a_{2}, \ldots \ldots \ldots \ldots \ldots \ldots, a_{P} \\ b_{1}+r, b_{2}, \ldots \ldots \ldots \ldots ., b_{q}\end{array}\right]$

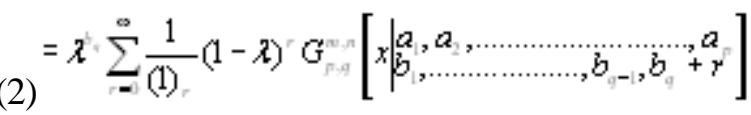

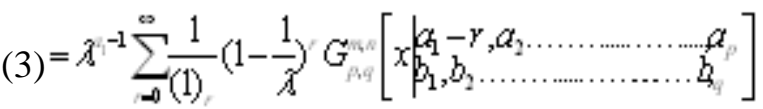

(4) $=\mathcal{A}^{-1} \sum_{r=0}^{\infty} \frac{1}{(1)_{r}}\left(\frac{1}{\lambda}-1\right)^{r} G_{p A}^{m+n}\left[x\left[\begin{array}{l}a_{1}, \ldots \ldots \ldots, a_{p-q}, a_{p}-r \\ l_{1}, b_{2} \ldots \ldots \ldots \ldots \ldots, b_{q}\end{array}\right]\right.$

The convergence conditions of $1.2(1)-1.2$ (4) are satisfied with Meijer's G-function defined in 1.1 (4).

Proof : To illustrate the proof of $1.2(1)-1.2(4)$, we shall prove 1.2 (1). For this, we replace G-function defined in 1.1 (4) on the right side of it by its equivalent Barnes' type of contour integral to obtain.

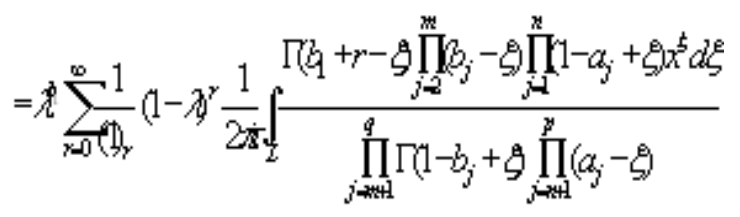

$\frac{1}{2 \pi} \int_{L} \frac{\Gamma\left(b_{1}-\xi\right) \prod_{j=1}^{m} \Gamma\left(b_{j}-\xi\right) \prod_{j=1}^{n}\left(1-a_{j}+\xi\right)}{\prod_{j=m+1}^{g} \Gamma\left(1-b_{j}+\xi\right) \prod_{j=n+1}^{n}\left(a_{j}-\xi\right)} x^{5} d \xi$,

since $\Gamma\left(b_{1}+r-\xi\right)=\Gamma\left(b_{1}-\xi\right)\left(b_{1}-\xi\right)_{r}$

Making use of the following Binomial summation :

$$
\sum_{\substack{n=0 \\ \text { we must have }}}^{\infty} \frac{(\alpha)_{n} x^{n}}{(1)_{n}}=(1-x)^{-\infty},
$$

$$
\lambda^{b_{1}} \sum_{r=0}^{\infty} \frac{\left(b_{1}-\xi\right)_{r}(1-\lambda)^{\gamma}}{(1)_{r}}=\lambda^{b_{1}} \lambda^{-b_{1}+\xi}=\lambda^{\xi}
$$

Thus, the ensuing result becomes

$\frac{1}{2 \pi} \int_{\frac{j}{2}} \frac{\prod_{j=1}^{m} \Gamma\left(b_{j}-\xi\right) \prod_{j=1}^{n} \Gamma\left(1-a_{j}+\xi\right)(\lambda x)^{\frac{5}{d}} d \xi}{\prod_{j=m+1}^{g} \Gamma\left(1-b_{j}+\xi\right) \prod_{j=n+1}^{n}\left(a_{j}-\xi\right)}$

$=G_{p, q}^{m, n}\left[\lambda x \mid \begin{array}{l}\left(a_{p}\right) \\ \left(b_{q}\right)\end{array}\right]$

Similarly, we can prove $1.2(2)-1.2$ (4) also.

\subsection{G-Functions of two variables}

The function defined by Agarwal (2005) give an extension of Meijer's

G-function to two variables and yields, as its special cases, the Appell's double hypergeometric function and Kampç dç Fçriçt hypergeometric function of higher order, besides all those functions which can be defined through G-symbol. Thus, the integral evaluated here includes a number of certain double series for generalized G-function of two variables.

Following Agarwal (2005), we define G-function of two variables by

$$
G_{p ;(r: k), q,(s: l)}^{n, n_{1}, n_{2}, m_{1}, m_{2}}\left[\begin{array}{c} 
\\
x \\
y \\
\left(c_{r}\right) ;\left(e_{k}\right) \\
\left(b_{q}\right) \\
\left(d_{s}\right) ;\left(f_{l}\right)
\end{array}\right]
$$

$=-\frac{1}{4 \pi^{2}} \int_{L_{1}} \int_{L_{2}} \phi(\xi+\eta) \psi(\xi, \eta) x^{5} y^{\eta} d \xi d \eta$,

where 
$\phi(\xi+\eta)=\Gamma\left[\begin{array}{c}1-\left(a_{n}\right)+\xi+\eta \\ \left(a_{n+1, y}\right)-\xi-\eta,\left(b_{q}\right)+\xi+\eta\end{array}\right]$,

$H(\xi \eta)=\left[\begin{array}{c}\left(d_{m}\right)-\xi\left(c_{n}\right)+\xi\left(f_{m}\right)-\eta\left(e_{n}\right)+\eta \\ 1-\left(d_{m+1}\right)+\xi 1-\left(c_{n+1 \eta}\right)-\xi 1-\left(f_{m+1}\right)+\eta 1-\left(e_{m+4 k}\right)-\eta\end{array}\right]$

The function 1.3 (1) holds for $|\mathrm{x}|<1,|\mathrm{y}|<1$ and $\mathrm{p}, \mathrm{q}, \mathrm{r}, \mathrm{s}, \mathrm{k}, \mathrm{l}, \mathrm{m}_{1}, \mathrm{~m}_{2, \mathrm{n}}, \mathrm{n}_{1}, \mathrm{n}_{2}$ are non-negative integers such that

$0 \leq \mathrm{n} \leq \mathrm{p}, 0 \leq \mathrm{n}_{1} \leq \mathrm{r}, 0 \leq \mathrm{n}_{2} \leq \mathrm{k}, 0 \leq \mathrm{m}_{1} \leq \mathrm{s}, 0 \leq \mathrm{m}_{2} \leq 1$, and for convergence, we have

$\mathrm{p}+\mathrm{q}+\mathrm{s}+\mathrm{r}<2\left(\mathrm{~m}_{1}+\mathrm{n}_{1}+\mathrm{n}\right)$

$\mathrm{p}+\mathrm{q}+1+\mathrm{k}<2\left(\mathrm{~m}_{2}+\mathrm{n}_{2}+\mathrm{n}\right)$,

$|\arg \mathrm{x}|<\pi\left[\mathrm{m}_{1}+n_{1}+n-\frac{1}{2}(p+q+s+r)\right]$, $\&|\arg \mathrm{y}|<\pi$

$\left[\mathrm{m}_{2}+n_{2}+n-\frac{1}{2}(p+q+l+k)\right]$.

Moreover, we shall mention further extension of the above hypergeometric series of two variables. Appell (1926), defined four functions $\mathrm{F}_{1}, \mathrm{~F}_{2}, \mathrm{~F}_{3}$ and $\mathrm{F}_{4}$ of two variables analogous to Gauss's function (i.e., series) ${ }_{2} \mathrm{~F}_{1}(\mathrm{a}, \mathrm{b} ; \mathrm{c} ; \mathrm{z})$ in the following form :

$\left(A_{1}\right) \quad F_{1}(a ; b, b ; c ; x, y)$

$=\sum_{m=0}^{\infty} \frac{(a)_{m+n}(b)_{m}\left(b^{\prime}\right)_{n} x^{m} y^{n}}{(1)_{m}(1)_{n}(c)_{m+n}}$

$\left(A_{2}\right) \quad F_{2}\left(a ; b, b ; c, c^{\prime} ; x, y\right)$

$=\sum_{m, n=0}^{\infty} \frac{(a)_{m+n}(b)_{m}\left(b^{\prime}\right)_{n} x^{m} y^{n}}{(1)_{m}(1)_{n}(c)_{m}\left(c^{\prime}\right)_{n}}$

$\left(\mathrm{A}_{3}\right) \quad \mathrm{F}_{3}(\mathrm{a}, \mathrm{a} ; \mathrm{b}, \mathrm{b} ; \mathrm{c} ; \mathrm{x}, \mathrm{y})$

$$
=\sum_{m, n=0}^{\infty} \frac{(a)_{m}\left(a^{\prime}\right)_{n}(b)_{m}\left(b^{\prime}\right)_{n} x^{m} y^{n}}{(1)_{m}(1)_{n}(c)_{m+n}}
$$

$\left(\mathrm{A}_{4}\right) \quad \mathrm{F}_{4}(\mathrm{a} ; \mathrm{b} ; \mathrm{c}, \mathrm{c} ; \mathrm{x}, \mathrm{y})$

$$
=\quad \sum_{m, n=0}^{\infty} \frac{(a)_{m+n}(b)_{m+n} x^{m} y^{n}}{(1)_{m}(1)_{n}(c)_{m}\left(c^{\prime}\right)_{n}} \text {. }
$$

All the above four double series are absolutely convergent for . $\mathrm{x} .<., \mathrm{y} .<1$;
$. \mathrm{x},+, \mathrm{y},<1$ and $. \mathrm{x}, \mathrm{y}, \frac{1}{2},<1$.

It is notable that all the Appell series shall reduce to the ordinary Gauss series (hypergeometric series) ${ }_{2} \mathrm{~F}_{1}$ (a, b; $c ; x)$ when $y=0$. The first three series shall also reduce to ${ }_{2} \mathrm{~F}_{1}(\mathrm{a}, \mathrm{b} ; \mathrm{c} ; \mathrm{x})$ when $\mathrm{b}=0$.

\subsection{Main Results}

The following double summation formula due to Carlitz (1967) shall be used:

$$
\begin{aligned}
& \sum_{m=0}^{\infty} \sum_{n=0}^{\infty} \frac{(a)_{m}\left(a^{\prime}\right)_{n}(b)_{m+n}(c)_{m}\left(c^{\prime}\right)_{n}}{(1)_{n}(b)_{m}(b)_{n}\left(c+c^{\prime}\right)_{m+n}}, \\
= & \Gamma\left[\begin{array}{l}
c-a^{\prime}, c^{\prime}-a, b, b-a-a^{\prime}, c+c^{\prime} ; \\
c+c^{\prime}-a-a^{\prime}, b-a, b-a^{\prime}, c, c^{\prime}
\end{array}\right] .
\end{aligned}
$$

We shall now establish the following results involving G-functions of two variables:

$$
\int_{0}^{1} \int_{0}^{1} \lambda^{c-1}(1-\lambda)^{c^{\prime}-1} \mu^{d^{-1}} \mu^{d^{\prime}-1} F_{2}\left[\begin{array}{l}
b ; a, a^{\prime} ; \\
\lambda, 1-\mu \\
-; b, b ;
\end{array}\right]
$$

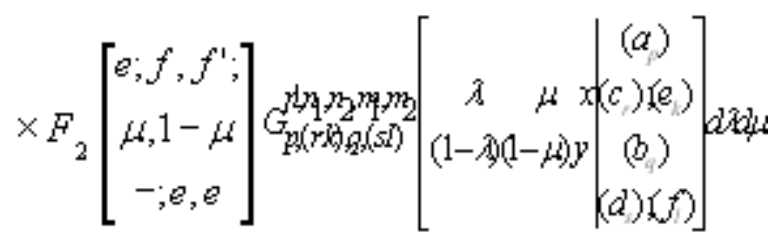

$$
=\Gamma\left[\begin{array}{l}
b, e, b-a-a^{\prime}, e-f-f^{\prime} \\
b-a, e-f, b-a^{\prime}, e-f^{\prime}
\end{array}\right]
$$

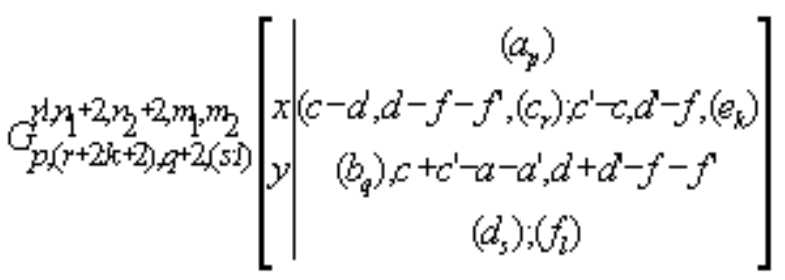

$$
\iint \lambda^{-1}(1-\lambda)-\mu^{d-1}(1-\mu)^{d-1} F_{2}\left[\begin{array}{l}
b ; a, a ; \\
\lambda, 1-\mu \\
-; b, b ;
\end{array}\right]
$$




$$
\begin{aligned}
& F_{2}\left[\begin{array}{c}
e ; f, f^{\prime} ; \\
\mu, 1-\mu \\
-; e, e ;
\end{array}\right] G_{p,(\gamma: k), q,(s: l)}^{n^{\prime}, n_{1}, n_{2}, m_{1}, m_{2}}\left[\begin{array}{c|c}
\left(a_{p}\right) \\
(1-\lambda)(1-\mu) x \\
\left(c_{r}\right) ;\left(e_{k}\right) \\
\left(b_{q}\right) \\
\left(d_{s}\right) ;\left(f_{l}\right)
\end{array}\right] d \lambda d \mu \\
& =\Gamma\left[\begin{array}{c}
b, e, c-a^{\prime}, b-a-a^{\prime}, d-f^{\prime}, e-f-f^{\prime} ; \\
b-a, e-f, b-a^{\prime}, e-f^{\prime}
\end{array}\right] \\
& \times \underset{p+2,(r: k), q+2,(s: l)}{G^{\prime}, n_{1}+2, n_{2}, m_{1}, m_{2}}\left[y \mid \begin{array}{c}
1-c^{\prime}+a, 1-a^{\prime}+f,\left(a_{p}\right) \\
\left(c_{r}\right) ;\left(e_{k}\right) \\
\left(b_{q}\right), c+c^{\prime}-a-a^{\prime}, d+d^{\prime}-f-f^{\prime} \\
\left(d_{s}\right) ;\left(f_{l}\right)
\end{array}\right]
\end{aligned}
$$

The convergence conditions of 1.4 (2) and 1.4 (3) hold for 1.3 (1).

\section{Proof}

We shall make use of Appell function $\mathrm{F}_{2}$ defind in $1.3\left(\mathrm{~A}_{2}\right)$ and replace G-function of two variables defined in 1.3 (1) on the left side of the result by its equivalent double Barnes' type of contour integrals to find.

$$
\begin{aligned}
& \iint_{0}^{1} \lambda^{c-1}(1-\lambda)^{c^{\prime}-1} \mu^{d-1}(1-\mu)^{d^{\prime}-1} \times \sum_{m, n=0}^{\infty} \frac{(b)_{m+n}(a)_{m}(a)_{n} \lambda^{m}(1-\lambda)^{n}}{(1)_{m}(1)_{n}(b)_{m}(b)_{n}} \\
& \times \sum_{r, s=0}^{\infty} \frac{(e)_{r+s}(f)_{r}\left(f^{\prime}\right)_{s} \mu^{r}(1-\mu)^{s}}{(1)_{r}(1)_{s}(e)_{r}(e)_{s}} d \lambda d \mu \\
& \times \frac{1}{(2 \pi)^{2}} \int_{L_{1} L_{2}} \phi(\xi+\eta) \Psi(\xi, \eta)(\lambda \alpha x)^{\xi}\{(1-\lambda)(1-\mu)\}^{n} d \xi d \eta \\
& =\int_{0}^{1} \lambda^{c-1+m+\xi}(1-\lambda)^{c^{\prime}-1+n+n} d \lambda \times \int_{0}^{1} \mu^{d-1+r+\xi}(1-\mu)^{d^{\prime}-1+s+n} d \mu \\
& \times \sum_{m, n} \frac{(b)_{m+n}(a)_{m}\left(a^{\prime}\right)_{n}}{(1)_{n}(b)_{m}(b)_{n}} \times \sum_{r, s} \frac{(e)_{r+s}(f)_{r}\left(f^{\prime}\right)_{s}}{(1)_{r}(1)_{s}(e)_{r}(e)_{s}} \times \frac{1}{(2 \pi)^{2}} \int_{L_{1}} \int_{L_{2}} \phi(\xi+\eta) \psi(\xi, \eta) x^{\xi} y^{\eta} d \xi d \eta
\end{aligned}
$$

interchanging the series term by term due to the absolute convergence and the integrals involved.

$$
=\frac{\Gamma(c+m+\xi) \Gamma\left(c^{\prime}+n+\eta\right)}{\Gamma\left(c+c^{\prime}+m+n+\xi+\eta\right)} \times \frac{\Gamma(d+r+\xi) \Gamma\left(d^{\prime}+s+\eta\right)}{\Gamma\left(d+d^{\prime}+r+s+\xi+\eta\right)}
$$




$$
\begin{aligned}
& \times \sum_{m, n} \frac{(b)_{m+n}(a)_{m}\left(a^{\prime}\right)_{n}}{(1)_{n}(b)_{m}(b)_{n}} \times \sum_{r, s} \frac{(e)_{r+s}(f)_{r}(f)_{s}}{(1)_{r}()_{s}(e)_{r}(e)_{s}} \times \frac{1}{(2 \pi)^{2}} \int_{L_{1} L_{2}} \int_{L} \not(\xi+\eta) \psi(\xi, \eta) x^{\xi} y^{\eta} d \xi \eta \eta \\
& \text { since } \int_{0}^{1} x^{m-1}(1-x)^{n-1} d x=\frac{\Gamma(m) \Gamma(n)}{\Gamma(m+n)} \text {. } \\
& =\sum_{m, n} \frac{(b)_{m+n}(a)_{m}\left(a^{\prime}\right)_{n}(c+\xi)_{m}\left(c^{\prime}+\eta\right)_{n}}{(b)_{m}(b)_{n}\left(c+c^{\prime}+\xi+\eta\right)_{m+n}} \times \sum_{\gamma_{s} s} \frac{(e)_{r+s}(f)_{r}\left(f^{\prime}\right)_{s}(d+\xi)_{r}\left(d^{\prime}+\eta\right)_{r}(e)_{s}\left(d+d^{\prime}+\xi+\eta\right)_{r+s}}{(d-\xi)} \\
& \times \frac{1}{(2 \pi)^{2}} \int_{2} \int_{L} \Gamma\left[\begin{array}{c}
c+\xi, c^{\prime}+\eta, d+\xi, d^{\prime}+\eta \\
c+c^{\prime}+\xi+\eta, d+d^{\prime}+\xi+\eta
\end{array}\right] \times \phi(\xi+\eta) \mu(\xi, \eta) x^{5} y^{\eta} d \xi \eta
\end{aligned}
$$

which yields the right side of (3) after summing the quadriple series with the help of 1.4 (1) and the proof is thus complete.

The proof of (2) can also be similarly established

\section{Special Cases}

Putting $\mathrm{p}=\mathrm{q}=0, \mathrm{~s}=1, \mathrm{r}=\mathrm{k}, \mathrm{m}_{2}=1, \mathrm{y}=0$ and $\mathrm{r} \leq \mathrm{s}, 1.4$ (2) implies that

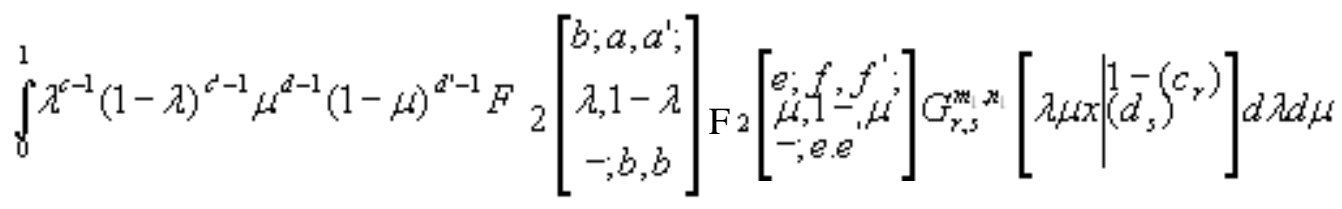

$$
\begin{aligned}
& =\Gamma\left[\begin{array}{c}
b, e, b-a-a^{\prime}, e-f-f^{\prime} ; \\
b-a, b-a, e-f, e-f^{\prime}
\end{array}\right] \times G_{\gamma+2, s+2}^{m, n+2}\left[x \mid 1-\left(c_{\gamma}\right) ; 1-c+a^{\prime}, 1-d+f^{\prime}\right], \\
& \text { provided that }|\mathrm{x}|<1,0 \leq \mathrm{m}_{1} \leq \mathrm{s}, 0 \leq \mathrm{n}_{1} \leq \mathrm{r}, \mathrm{r}+\mathrm{s}<2\left(\mathrm{~m}_{1}+\mathrm{n}_{1}\right) \\
& \text { and }|\arg x|<\pi\left[m_{1}+n_{1}-\frac{1}{2}(r+s)\right], \operatorname{Re}\left(\mathrm{c}, \mathrm{c}^{\prime}, \mathrm{d}, \mathrm{d}^{\prime}\right)>0 \text {. }
\end{aligned}
$$

Similarly, we can deduce the other cases for G-function of one variable of 1.4 (3) also. 


\section{Acknowledgement}

I am grateful to Dr. R.M. Shrestha and Dr. S.R. Pant, Central Department of Mathematics, Tribhuvan University for their kind inspiraction and encouragement to me in the preparation of this paper.

\section{References}

Agarwal, R.P. 1965. An extension of Meijer's G-function. Proc. Nat. Inst. Sci. 31: 536-546.

Agarwal, R.P. H.L.Manocho, and K.S. Rao 2001. Recent development in the theory of generalized hyper geometric series. In: Selected topics in special functions. Allied Publishers Ltd. New Delhi. pp. 1-20.

Appell, P. and Kampç dç Fçriet. 1926. Functions hypergeometriques at hypersphereques. Gauthiervillars, Paris.
Bailey, W.N. 1935. Generalized hypergeometric series. Cambridge University Press, London.

Carlitz, L. 1967. A summation theorem for double hypergeometric series. Rendicontidies seninario mathematico della universitadi padova. pp. 230-233.

Denis, R.Y. 1972. Certain expansions of generalized hypergeometric series. The Math. Stud. XI A. pp. $82-86$.

Gasper, G. and M. Rahman 1990. Basic hypergeometric series. Cambridge Univ. Press, London.

Meijer, C.S. 1936. Uber Whittakerche bezw, Besselsche funktionen and deren produkte. Nieuw Arch. Wiskunde 18 (4): 10-39.

Sharma, B.R. 2000. On certain generating results for $\mathrm{H}-$ functions, Journal of Purwanchal Academy of Science. 6: 72-77. 
Nepal Journal of Science and Technology 9 (2008) 